\title{
BMJ Open Educational differences in psychological distress? Results from a population- based sample of men and women in Sweden in 2012
}

\author{
Anu Molarius, ${ }^{1,2}$ Fredrik Granström ${ }^{3}$
}

To cite: Molarius A, Granström F. Educational differences in psychological distress? Results from a population-based sample of men and women in Sweden in 2012. BMJ Open 2018;8:e021007. doi:10.1136/ bmjopen-2017-021007

- Prepublication history for this paper is available online. To view these files, please visit the journal online (http://dx.doi. org/10.1136/bmjopen-2017021007).

Received 7 December 2017

Revised 2 March 2018

Accepted 9 April 2018
Check for updates

${ }^{1}$ Competence Centre for Health, Region Västmanland, Vasteras, Sweden

${ }^{2}$ Department of Public Health Sciences, Karlstad University, Karlstad, Sweden

${ }^{3}$ Division of Community Medicine, Department of Medical and Health Sciences, Linköping University, Linköping, Sweden

Correspondence to Dr Anu Molarius; anu.molarius@ regionvastmanland.se

\section{ABSTRACT}

Background Mental health problems are more frequent in socially disadvantaged groups, but the results vary between different studies, different populations and different measures of mental health. This paper investigated the association between educational level, economic difficulties and psychological distress in men and women in Sweden.

Methods The study population included 24510 respondents aged 25-74 years who responded to a survey questionnaire in Mid-Sweden in 2012 (response rate 53\%). Psychological distress was measured with the 12-item version of the General Health Questionnaire, and multivariate logistic regression models were used in statistical analyses, adjusting for age, employment status and social support.

Results The prevalence of psychological distress was higher in women $(16.4 \%)$ than in men $(11.3 \% ; p<0.001)$. Persons with low and medium educational level had a lower risk of psychological distress than persons with high educational level after adjustment for confounders. Economic difficulties had a strong association with psychological distress (OR $2.80(95 \% \mathrm{Cl} 2.39$ to 3.27$)$ and OR $2.40(95 \% \mathrm{Cl} 2.12$ to 3.71$)$ in men and women, respectively) after adjustment for confounders.

Conclusion We found a strong association between economic difficulties and psychological distress in this study, but no inverse association between educational level and psychological distress. On the contrary, persons with high education had more psychological distress than persons with low and medium education when age, employment status and social support were taken into account. The findings were similar in men and women.

\section{INTRODUCTION}

Social inequalities in health are well documented and have been found for several health outcomes such as mental health problems, ${ }^{1-3}$ self-rated health ${ }^{45}$ and mortality. The most often used measures of socioeconomic status include education, income and occupation. Mental health problems are the leading cause of disability worldwide and have large economic and social consequences for both the individual and society.

\section{Strengths and limitations of this study}

- The study is based on a considerable, representative sample of the general population in a large geographical area and covers a wide age group of men and women from 25 to 74 years.

- Internationally validated questionnaire (12-item version of the General Health Questionnaire) was used to measure psychological distress and register data to measure educational level.

- As people with poor health and/or low education often have lower response rates, the response rate of $53 \%$ may have led to underestimation or overestimation of the differences in mental health.

- The study is based on cross-sectional data which do not allow interpretations of the direction of the observed associations.

For mental health problems, there is strong evidence that low socioeconomic position is associated with severe mental health disorders such as major depression. ${ }^{89}$ Previous studies have also shown that less severe mental health problems are significantly more frequent in socially disadvantaged groups, such as the unemployed and persons with low education, low income and poor material standard of living. ${ }^{1}$

Even though low education is consistently associated with mental health problems, ${ }^{1}$ some previous studies have shown stronger associations with income or financial difficulties or strain than with educational level. ${ }^{2} 10-12$ Some studies have even found that the association is stronger for financial difficulties than for income. ${ }^{211}$ It has been suggested that the egalitarian socioeconomic policies in the Nordic countries may reduce the effect of income on health. ${ }^{11}$ However, also a longitudinal study in Britain found that financial strain in form of self-reported economic difficulties is a better independent predictor for future psychiatric morbidity than poverty and unemployment. ${ }^{13}$ In addition, differences in 
the association between educational level and mental health problems in the form of psychological distress between men and women have been reported. ${ }^{3}$ The associations may therefore differ between populations, genders and national or cultural contexts and may also change over time.

Socioeconomic differences in minor mental health problems are usually examined using validated and reliable measures such as the General Health Questionnaire (GHQ), 36-item Short Form Health Survey (SF-36) Mental Health Component Summary or the Fifth Question of the European Quality of Life Instrument (EQ-5D). GHQ measures psychological distress, which includes depressive symptoms, anxiety and affective well-being. ${ }^{14}$ It is a widely used measure of minor psychiatric morbidity, which primarily reflects general, non-psychotic and context-free mental health problems in the recent past but is also predictive of more severe mental disorders. ${ }^{14}$

A longitudinal study in Sweden investigated the associations between socioeconomic status and the risk of psychological distress and depression. ${ }^{12}$ The results showed that education was unrelated to either outcome among men and women, whereas income was associated with the risk of both outcomes and that the strength of the associations increased with symptom severity. The role of financial difficulties was not assessed in that study. A cross-sectional study based on the national public health survey in Sweden assessed the association between economic hardship and mental health problems, but the independent effect of educational level was not studied. ${ }^{11}$

The aim of this study was therefore to investigate the association between educational level, economic difficulties and mental health problems in the form of psychological distress in a population-based sample in Sweden in 2012. Also, possible differences in these associations between men and women were assessed.

\section{METHODS}

The study is based on a survey questionnaire sent to a random population sample in four counties in Mid-Sweden in 2012. The survey was carried out in collaboration with the Public Health Institute (now the Public Health Agency of Sweden) and the four counties (Uppsala, Sörmland, Västmanland and Örebro). The age range was $25-74$ years, and the study population included 24510 respondents (response rate 53\%).

Information on gender, age and educational level was based on register data from Statistics Sweden. Levels of education were categorised into compulsory school or equivalent education for 9 years or less, secondary education (10-12 years of education) and postsecondary education (more than 12 years of education). Current economic difficulties were assessed by asking whether the respondent had had problems with current expenditure for food, rent, bills and so on during the past 12 months (yes/no).
Psychological distress was measured by the 12-item version of the GHQ (GHQ-12). ${ }^{14}$ A recommended and commonly used cut-off point of three or more symptoms was used in this study to indicate psychological distress. ${ }^{211} 14$

Since employment status and social support are associated with mental health problems, ${ }^{10}$ we considered them as potential confounders. Employment status was derived from a survey question about whether the respondent was employed, self-employed, student, unemployed, on sickness leave (more than 3 months), on disability pension or retired. Social support was assessed by the question: 'Do you have anyone you can share your innermost feelings with and confide in?' (yes/no).

The respondents gave their informed consent for applying the registry data by answering the questionnaire. After the record linkage, all identity information was removed before the material was handed over from Statistics Sweden to the county councils. Permission from the regional ethical review board in Uppsala has been obtained (EPN 2012/256).

\section{Patient and public involvement}

The study did not involve patients. The results of the survey are disseminated to the public through websites of the four county councils.

\section{Statistical methods}

Prevalence of psychological distress is reported by educational level and economic difficulties in men and women. Multivariate logistic regression models were used to examine the association between educational level, economic difficulties and psychological distress. Separate models were run for men and women. Since the prevalence of mental health problems is higher in younger age groups, ${ }^{8}$ all the models were adjusted for age (in 10-year groups). The first model only included age and educational level as independent variables. In the second model, economic difficulties were added. In the final model, the potential confounders employment status and social support were also included. The results are reported as ORs and 95\% CIs for psychological distress. SPSS V.24 was used for all analyses.

\section{RESULTS}

About half of the study population had medium educational level (table 1). About 1 in 10 had only compulsory education, and $32 \%$ of the men and $40 \%$ of the women had postsecondary education. Overall, $12 \%$ of the men and $15 \%$ of the women reported economic difficulties during the last 12 months.

The prevalence of psychological distress was higher in women $(16.4 \%)$ than in men $(11.3 \%$; $\mathrm{p}<0.001)$. There were rather small differences between educational levels, with somewhat higher levels of psychological distress at high educational level (table 2). There was, however, a strong association between economic 
Table 1 Characteristics of the study population, men and women aged 25-74 years in 2012

\begin{tabular}{lll}
\hline Variable & Men & Women \\
\hline $\mathrm{n}$ & 11133 & 13377 \\
\hline Age (mean, SD) & $55.4(13.5)$ & $53.4(13.8)$ \\
\hline Educational level (\%) & & \\
$\quad$ Low & 20.5 & 15.3 \\
$\quad$ Medium & 47.6 & 44.8 \\
$\quad$ High & 31.9 & 39.9 \\
\hline Economic difficulties (\%) & & \\
$\quad$ No & 88.4 & 85.4 \\
\hline Yes & 11.6 & 14.6 \\
\hline Employment status (\%) & & \\
$\quad$ Employed & 52.0 & 56.8 \\
\hline Self-employed & 10.6 & 4.1 \\
\hline Student & 1.4 & 2.9 \\
$\quad$ Unemployed & 2.9 & 3.5 \\
\hline On sickness leave & 1.9 & 3.4 \\
\hline On disability pension & 2.8 & 4.4 \\
\hline Retired & 28.3 & 24.9 \\
\hline Social support (\%) & & \\
\hline Yes & 87.7 & 90.9 \\
\hline No & 12.3 & 9.1 \\
\hline
\end{tabular}

difficulties and psychological distress in both men and women.

In the age adjusted model, the OR for psychological distress for the low educational level was not statistically significant when compared with the high educational level (table 2). Persons with medium educational level had a lower risk of psychological distress than persons with high educational level. Further adjustment for economic difficulties showed that economic difficulties had a strong association with psychological distress, but the association between educational level and psychological distress remained practically unchanged. Further adjustment for employment status and social support attenuated somewhat the OR for economic difficulties, but the association remained strong and statistically significant. The OR for psychological distress at low educational level became statistically significantly below one. No statistically significant differences were observed in the ORs for educational level and economic difficulties between men and women.

\section{DISCUSSION}

The findings of this study suggest that there is no inverse association between educational level and psychological distress in this adult population in Sweden. A strong association between economic difficulties and psychological distress was, however, found. The associations between educational level, economic difficulties and psychological

Table 2 Prevalence and ORs from multivariate logistic regression models for psychological distress by educational level and current economic difficulties in men and women aged 25-74 years in 2012

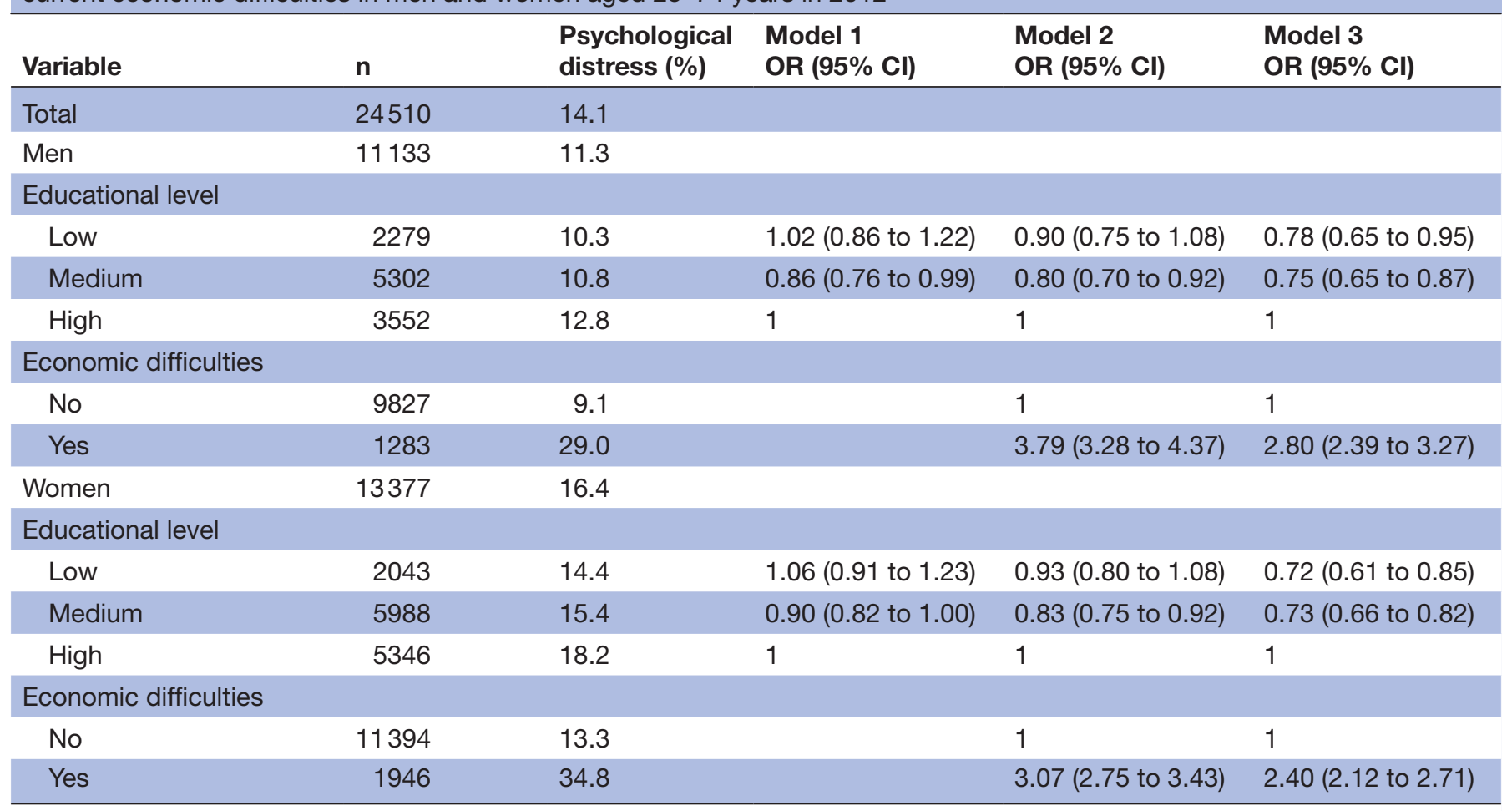

Model 1: adjusted for age.

Model 2: adjusted for age and economic difficulties.

Model 3: adjusted for age, economic difficulties, employment status and social support. 
distress, respectively, were rather similar in men and in women even though the prevalence of psychological distress was higher among women.

Several previous studies have shown educational differences in mental health problems, ${ }^{1}$ but a weak association between educational level and common mental disorders has been observed, for example, in a working population in Finland. ${ }^{2}$ No association between low education and mental health problems has been reported from some studies in Sweden, ${ }^{10}$ whereas a Danish study showed a relationship between low education and high prevalence of both minor and major depression..$^{15} \mathrm{~A}$ strong association between low education and clinical depression has also been reported among representative population-based studies conducted in Finland, Poland and Spain. ${ }^{16}$ These findings suggest that low education may be more consistently related to severe mental health problems than to minor psychiatric morbidity.

The results concerning economic difficulties are in line with a national Swedish study where economic hardship was strongly associated with psychological distress. ${ }^{11}$ Several other studies have also shown stronger associations with income or financial difficulties or strain than with educational level. ${ }^{2}{ }^{1012}$ Of the measures of socioeconomic positions, educational level is usually the most distant and acquired first. It affects occupation and employment status which in turn contribute to income. Income contributes to material standards of living and purchasing power. Yet, economic difficulties reflect more proximate and accumulated influences or may be more related to adverse changes in living conditions which may contribute to their stronger association with mental health problems. ${ }^{1} 21113$

The association between educational level and psychological distress was similar in men and women in our study. This is in line with several previous studies ${ }^{212}$ and does not confirm the gender differences found, for example, in Canada. ${ }^{3}$ Also, the association between economic difficulties and psychological distress was similar in both genders reconfirming the findings from some previous studies. ${ }^{211}$

The current study has some limitations. The response rate was $53 \%$. As people with poor health and/or low education often have lower response rates, this may lead to underestimation of educational differences in mental health. Also, an overestimation of the association is possible. ${ }^{17}$ Nevertheless, the strong association between economic difficulties and psychological distress suggests that the results should not be severely underestimated. In addition, the study is based on cross-sectional data which prevent any interpretations of the direction of the results. It is possible that mental health problems lead to reduced educational attainment, reduced income and higher level of economic difficulties. Previous longitudinal studies have, however, shown that the main direction is from income and economic difficulties to mental health problems. ${ }^{9} 1213$

In our analysis, we adjusted for employment status and social support in the final model. The association between economic difficulties and psychological distress was somewhat attenuated after this adjustment, especially among men, indicating that a part of the association may be explained by increased economic difficulties due to unemployment, long-term sickness leave or disability pension, but the association still remained strong after the adjustment in both men and women. On the other hand, after full adjustment, the OR for low educational level became statistically significantly below one indicating that persons with low education have less psychological distress than persons with high education when employment status and social support are taken into account. Data on some other potential confounders such as personal history of mental health problems were not available, and therefore, some residual confounding is possible. Furthermore, a common method bias may have affected the association between economic difficulties and psychological distress since both the exposure and outcome were self-reported. This, however, is more likely to lead to an underestimation than to an overestimation of the association. ${ }^{18}$

We used one measure of mental health problems, psychological distress (GHQ-12), in this study. Some previous studies have combined several measures. Lahelma $e t a l^{2}$ used both GHQ-12 and SF-36 mental component summary to examine the association between socioeconomic position and common mental disorders in their study among Finnish employees and found similar results for both measures as well as two different cut-off points for GHQ-12 indicating severity. Ahnquist and Wamala ${ }^{11}$ used three different indicators of mental health problems in their study of the Swedish population: psychological distress (GHQ-12), severe anxiety and use of antidepressant medication. Even they found that the association between economic hardship and mental health problems was unrelated to the indicator used.

One of the advantages of the present study is that it is based on a considerable, representative sample of the general population in a large geographical area and covers a wide age group of men and women from 25 to 74 years. Although the study was limited to four counties, it covers the general adult population in these counties, comprising almost 1 million inhabitants. The prevalence of psychological distress was similar to the national average in Sweden. ${ }^{19}$ Another strength is the use of GHQ-12 as a measure of psychological distress as it has been internationally validated. ${ }^{14}$ GHQ-12 has been used in several other studies to measure social inequalities in mental health problems. ${ }^{211}{ }^{12}$ Similar results as for GHQ-12 have also been reported for other measures of mental health problems such as the mental component summary of the SF-36 ${ }^{2}$ and the EQ-5D. ${ }^{10}$ Educational level, age and gender were based on register data which increase the reliability of these variables.

Mental health problems are the leading cause of disability worldwide, ${ }^{7}$ and social inequalities in health are a major public health challenge. In Sweden, there are large educational differences in self-rated health. Persons 
with low educational level have approximately twice as often poor self-rated health as persons with high educational level. ${ }^{520}$ As mental health problems are strongly associated with self-rated health, ${ }^{21}$ mental health problems may have a mediating role in the association between education and self-rated health. In this study, no inverse association between educational level and psychological distress was, however, found. It is therefore improbable that mental health problems would explain educational inequalities in self-rated health. The notion is further supported by the fact that psychosocial factors have a weaker contribution to educational inequalities in selfrated health than material factors. ${ }^{22}$ In this study, persons with high education had more psychological distress than persons with low and medium education after adjustment for confounders. Further studies are required in order to explain the positive association between educational level and psychological distress.

In summary, no inverse association between educational level and psychological distress was found in this study. Persons with medium educational had a somewhat lower level of psychological distress than persons with high education. After adjustment for employment status and social support, even persons with low educational level had less psychological distress than those with high education. However, a strong association between economic difficulties and psychological distress was found. The associations between educational level, economic difficulties and psychological distress did not differ between men and women.

Contributors AM conceived the study and analysed the data. Both authors interpreted the results, drafted and critically revised the manuscript and read and approved the final manuscript.

Funding The survey was funded by the County Councils of Västmanland (now Region Västmanland), Sörmland, Uppsala and Örebro.

Competing interests None declared.

Patient consent Not required.

Ethics approval The survey was approved by the regional ethical review board in Uppsala (EPN 2012/256).

Provenance and peer review Not commissioned; externally peer reviewed.

Data sharing statement № additional data available.

Open Access This is an Open Access article distributed in accordance with the Creative Commons Attribution Non Commercial (CC BY-NC 4.0) license, which permits others to distribute, remix, adapt, build upon this work non-commercially, and license their derivative works on different terms, provided the original work is properly cited and the use is non-commercial. See: http://creativecommons.org/ licenses/by-nc/4.0/

(C) Article author(s) (or their employer(s) unless otherwise stated in the text of the article) 2018. All rights reserved. No commercial use is permitted unless otherwise expressly granted.

\section{REFERENCES}

1. Fryers $T$, Melzer D, Jenkins R. Social inequalities and the common mental disorders: a systematic review of the evidence. Soc Psychiatry Psychiatr Epidemiol 2003;38:229-37.

2. Lahelma E, Laaksonen M, Martikainen P, et al. Multiple measures of socioeconomic circumstances and common mental disorders. Soc Sci Med 2006;63:1383-99.

3. Duchaine CS, Ndjaboué R, Levesque M, et al. Psychosocial work factors and social inequalities in psychological distress: a populationbased study. BMC Public Health 2017;17:91.

4. Eikemo TA, Bambra C, Joyce K, et al. Welfare state regimes and income-related health inequalities: a comparison of 23 European countries. Eur J Public Health 2008;18:593-9.

5. Hu Y, van Lenthe FJ, Borsboom GJ, et al. Trends in socioeconomic inequalities in self-assessed health in 17 European countries between 1990 and 2010. J Epidemiol Community Health 2016;70:644-52.

6. Mackenbach JP, Kulhánová I, Artnik B, et al. Changes in mortality inequalities over two decades: register based study of European countries. BMJ 2016;353:11732.

7. Whiteford HA, Degenhardt L, Rehm J, et al. Global burden of disease attributable to mental and substance use disorders: findings from the Global Burden of Disease Study 2010. Lancet 2013;382:1575-86.

8. Lorant $\mathrm{V}$, Deliège $\mathrm{D}$, Eaton $\mathrm{W}$, et al. Socioeconomic inequalities in depression: a meta-analysis. Am J Epidemiol 2003;157:98-112.

9. Wang JL, Schmitz N, Dewa CS. Socioeconomic status and the risk of major depression: the Canadian National Population Health Survey. J Epidemiol Community Health 2010;64:447-52.

10. Molarius A, Berglund K, Eriksson C, et al. Mental health symptoms in relation to socio-economic conditions and lifestyle factors-a population-based study in Sweden. BMC Public Health 2009;9:302.

11. Ahnquist J, Wamala SP. Economic hardships in adulthood and mental health in Sweden. The Swedish National Public Health Survey 2009. BMC Public Health 2011;11:788.

12. Kosidou K, Dalman C, Lundberg M, et al. Socioeconomic status and risk of psychological distress and depression in the Stockholm Public Health Cohort: a population-based study. J Affect Disord 2011;134:160-7.

13. Weich S, Lewis G. Poverty, unemployment, and common mental disorders: population based cohort study. BMJ 1998;317:115-9.

14. Goldberg DP, Gater R, Sartorius N, et al. The validity of two versions of the GHQ in the WHO study of mental illness in general health care. Psychol Med 1997;27:191-7.

15. Andersen I, Thielen K, Nygaard E, et al. Social inequality in the prevalence of depressive disorders. J Epidemiol Community Health 2009;63:575-81.

16. Freeman A, Tyrovolas S, Koyanagi A, et al. The role of socioeconomic status in depression: results from the COURAGE (aging survey in Europe). BMC Public Health 2016;16:1098.

17. Hernán MA, Hernández-Díaz S, Robins JM. A structural approach to selection bias. Epidemiology 2004;15:615-25.

18. Rothman K, Greenland S, Last TL. Modern epidemiology. 3rd edn. Philadelphia: Lippincott Williams and Wilkins, 2008.

19. The Public Health Agency of Sweden. Hälsa på lika villkor? Nationella folkhälsoenkäten. [Health on equal terms? The national public health survey]. www.folkhalsomyndigheten.se (accessed 22 Feb 2018).

20. Granström F, Molarius A, Garvin P, et al. Exploring trends in and determinants of educational inequalities in self-rated health. Scand $J$ Public Health 2015;43:677-86.

21. Molarius A, Janson S. Self-rated health, chronic diseases, and symptoms among middle-aged and elderly men and women. J Clin Epidemiol 2002;55:364-70.

22. Moor I, Spallek J, Richter M. Explaining socioeconomic inequalities in self-rated health: a systematic review of the relative contribution of material, psychosocial and behavioural factors. J Epidemiol Community Health 2017;71:565-75. 